\title{
Effects of resistant starch on glycemic control, serum lipoproteins and systemic inflammation in patients with metabolic syndrome and related disorders: A systematic review and meta-analysis of randomized controlled clinical trials
}

\author{
Jamal Halajzadeh ${ }^{\mathrm{a}}$, Alireza Milajerdi ${ }^{\mathrm{b}, \mathrm{c}}$, Željko Reiner ${ }^{\mathrm{d}}$, Elaheh Amirani ${ }^{\mathrm{e}}$, Fariba Kolahdooz ${ }^{\mathrm{f}}$, Maryam Barekat ${ }^{\mathrm{g}}$, \\ Hamed Mirzaei ${ }^{\mathrm{e}}$, Seyyed Mehdi Mirhashemi ${ }^{\mathrm{h}}$, and Zatollah Asemi ${ }^{\mathrm{e}}$ \\ ${ }^{a}$ Department of Biochemistry and Nutrition, Research Center for Evidence-Based Health Management, Maraghe University of Medical \\ Science, Maraghe, Iran; 'bStudents' Scientific Research Center, Tehran University of Medical Sciences, Tehran, Iran; 'Department of \\ Community Nutrition, School of Nutritional Sciences and Dietetics, Tehran University of Medical Sciences, Tehran, Iran; dDepartment of

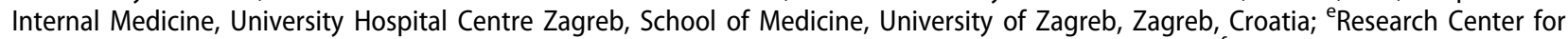 \\ Biochemistry and Nutrition in Metabolic Diseases, Kashan University of Medical Sciences, Kashan, Iran; Indigenous and Global Health \\ Research, Department of Medicine, University of Alberta, Edmonton, Canada; ${ }^{9}$ Department of Regenerative Biomedicine, Cell Science \\ Research Center, Royan Institute for Stem Cell Biology and Technology, ACECR, Tehran, Iran; ${ }^{\text {h}}$ Metabolic Diseases Research Center, Research \\ Institute for Prevention of Non-Communicable Diseases, Qazvin University of Medical Sciences, Qazvin, Iran
}

\begin{abstract}
The aim of this systematic review and meta-analysis was to evaluate the effects of resistant starch (RS) on glycemic status, serum lipoproteins and inflammatory markers in patients with metabolic syndrome (MetS) and related disorders. Two independent authors systematically searched online database including EMBASE, Scopus, PubMed, Cochrane Library, and Web of Science from inception until 30 April 2019. Cochrane Collaboration risk of bias tool was applied to assess the methodological quality of included trials. The heterogeneity among the included studies was assessed using Cochrane's $Q$ test and I-square $\left(\mathrm{I}^{2}\right)$ statistic. Data were pooled using a random-effects model and weighted mean difference (WMD) was considered as the overall effect size. Nineteen trials were included in this meta-analysis. Administration of RS resulted in significant reduction in fasting plasma glucose (FPG) (14 studies) (WMD: $-4.28 ; 95 \% \mathrm{Cl}:-7.01,-1.55)$, insulin (12 studies) (WMD: $-1.95 ; 95 \% \mathrm{Cl}:-3.22,-0.68)$, and HbA1C (8 studies) (WMD: $-0.60 ; 95 \% \mathrm{Cl}:-0.95,-0.24)$. When pooling data from 13 studies, a significant reduction in total cholesterol levels (WMD: $-8.19 ; 95 \%$ $\mathrm{Cl}:-15.38,-1.00)$ and LDL-cholesterol (WMD: $-8.57 ; 95 \% \mathrm{Cl}:-13.48,-3.66)$ were found as well. Finally, RS administration was associated with a significant decrease in tumor necrosis factor alpha (TNF- $\alpha$ ) (WMD: $-2.02 ; 95 \% \mathrm{Cl}:-3.14,-0.90)$. This meta-analysis showed beneficial effects of RS on improving FPG, insulin, HbA1c, total cholesterol, LDL-cholesterol and TNF- $\alpha$ levels in patients with MetS and related disorders, but it did not affect HOMA-IR, triglycerides, HDL-cholesterol, CRP and IL-6 levels.
\end{abstract}

\section{KEYWORDS}

Resistant starch; insulin resistance; metabolic syndrome; LDL-cholesterol; HDL-cholesterol; meta-analysis

\section{Introduction}

Currently, growing evidence suggests that intestinal microflora plays as important role in the development of metabolic disorders such as dyslipidemia, insulin resistance and inflammation (Panwar et al. 2013; Shen, Obin, and Zhao 2013). Microflora is defined as microorganisms that live within the human bodies (more than 2000 species of commensal bacterial organisms) (Neish 2009). Digestive tract is the most important part of the body where these microorganisms, especially bacteria, live. Many microorganisms, mostly gut microflora, have an influence on the function of human body by different mechanisms such as immunomodulation, protection by formation of physical barriers, antiinflammatory by production of short chain fatty acids
(SCFA), and production of bacteriocins (Montalto et al. 2009). Changes in composition of gut microflora can have beneficial or unfavorable effects on the health of the host organism. Diet is one of many factors that can cause persistence of a specific bacteria in the digestive tract (Panwar et al. 2013; Sekirov et al. 2010).

Resistant starch (RS) is defined as "selectively fermented ingredients that result in specific changes in the composition and/or activity of the gastrointestinal microbiota, thus conferring benefits upon host health" (Gibson et al. 2010). RS is a fraction of starch which is resistant to digestive enzymes in small intestine and is delivered intact to colon to be fermented by bacteria. RS divided into five subtypes (RS1-RS5) based on their structure and sources (Homayouni et al.

CONTACT Zatollah Asemi asemi_r@yahoo.com $B$ Research Center for Biochemistry and Nutrition in Metabolic Diseases, Kashan University of Medical

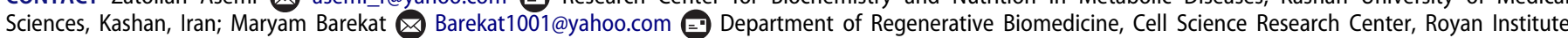
for Stem Cell Biology and Technology, ACECR, Tehran, Iran.

Color versions of one or more of the figures in the article can be found online at www.tandfonline.com/bfsn.

(C) 2019 Taylor \& Francis Group, LLC 